\title{
The effectiveness and value of digital health technologies as an adjunct to medication-assisted therapy for opioid use disorder
}

\author{
A summary from the Institute for Clinical and Economic Review's Midwest Comparative \\ Effectiveness Public Advisory Council
}

Jeffrey A Tice, MD; Melanie D Whittington, PhD; Jonathan D Campbell, PhD; and Steven D Pearson, MD, MSc

Opioid use disorder (OUD) is a public health crisis in the United States. The number of U.S. drug overdose deaths increased continuously from 1999 to mid-2017, ${ }^{1}$ when it reached a plateau of approximately 70,000 deaths per year, of which approximately 50,000 were from opioids. ${ }^{2,3}$ Sadly, during the COVID-19 epidemic, the number of overdose deaths has again begun to increase. ${ }^{4}$ The effect of the opioid crisis is felt financially, as well: the White House Council of Economic Advisors estimates that the opioid epidemic cost the United States $\$ 686$ billion in 2018 and more than $\$ 2.4$ trillion from 2015 to $2018 .{ }^{5}$

Medication-assisted treatment (MAT) is the most effective treatment for OUD, but more than half of patients starting MAT drop out of treatment within 3 to 6 months. ${ }^{6}$ Behavioral therapies have been shown to increase retention in some studies, but they are highly resource intensive. Digital health technologies (DHTs) offer the potential to expand the availability of behavioral therapies and to reduce cost. We examine the evidence for 3 such promising DHTs in this review: reSET-O, Connections, and DynamiCare.
DHTs for OUD offer the potential to enhance access to evidence-based MAT for patients whose schedules or locations present challenges to inperson appointments or for whom in-person appointments raise concerns about the stigma attached to this condition. The 3 DHTs evaluated in our review are all apps but differ in important ways. The first, reSET-O, is a 12-week prescription digital therapeutic that combines contingency management (CM) with OUD-specific cognitive behavioral therapy (CBT). The CM component of reSET-O gives small rewards (cash, gift cards) for desired behaviors (negative urine drug screen tests, completing CBT modules), and the size of the potential reward increases, on average, with consecutive desired behaviors. ${ }^{7}$

The second intervention, Connections, is an app that combines a form of digital CBT (CBT4CBT) with a program that enhances patient communication with addiction experts, peer support groups, and counselors (A CHESS). The third DHT, the DynamiCare app, includes CBT with CM, substance use screening results, Bluetooth-enabled breathalyzer for alcohol testing, drug saliva testing, and appointment monitoring and reminders.

\author{
Author affiliations \\ Jeffrey A Tice, MD, Division of General \\ Internal Medicine, University of \\ California, San Francisco. Melanie D \\ Whittington, PhD; Jonathan D Campbell, \\ $\mathrm{PhD}$; and Steven D Pearson, MD, MSc, \\ Institute for Clinical and Economic \\ Review, Boston, MA. \\ AUTHOR CORRESPONDENCE: \\ Jeffrey A Tice, jeff.tice@ucsf.edu \\ J Manag Care Spec Pharm. \\ 2021;27(4):528-32 \\ Copyright $\odot 2021$, Academy of Managed \\ Care Pharmacy. All rights reserved.
}

The Institute for Clinical and Economic Review (ICER) conducted a systematic literature review and cost-effectiveness analysis to evaluate the health and economic outcomes of DHTs for OUD. Complete details of ICER's systematic literature search and protocol, as well as the methodology and model structure for the economic evaluation are available on ICER's website. In this review, we present the summary of our findings 
and highlights of the policy discussion with key stakeholders held at a public meeting of the Midwest Comparative Effectiveness Public Advisory Council on November 20, 2020. The detailed report is available on the ICER website at https://icer.org/wp-content/uploads/2020/08/ICER_ DHTs_for_OUD_Final_Evidence_Report_121120-1.pdf.

\section{Summary of Findings}

\section{CLINICAL EFFECTIVENESS}

The most important clinical benefit reported in the trials is retention in MAT. Long-term retention (6 months to 2 years or longer) is associated with abstinence and with the outcomes that really matter to patients: employment, reduced financial stress, decreased hospitalizations and emergency room visits, and improved relationships. ${ }^{8-10}$ We found that none of the studies of these apps has any data on the outcomes that matter to patients, and earlier studies of the components of these DHTs do not show improvements in long-term retention.

In the evidence base available before our draft report, we found no randomized trials, cohort studies, or case series that evaluated any of the DHTs reviewed in this report. The 510(k) clearance of reSET-O by the U.S. Food and Drug Administration (FDA) was based on its similarity to reSET, a web-based precursor to reSET-O. The trial evaluating reSET involved participants who met the Diagnostic and Statistical Manual of Mental Disorders, Fourth Edition (DSM-4) criteria for opioid dependence and the FDA qualification criteria for buprenorphine treatment. ${ }^{11}$ There was no significant difference in the primary outcome: number of days of continuous abstinence at 12 weeks. Any other significant findings should therefore be considered hypothesis generating. The study did find a reduced likelihood of dropping out of treatment at 12 weeks ( $20 \%$ vs. $36 \%$, $\mathrm{HR}=0.47 ; 95 \% \mathrm{CI}=0.26-0.85)$ as compared with those who only received contingency management (CM) in addition to MAT. However, a study of the same intervention with 1-year follow-up found no difference in drop-out rates (38.8\% vs. $38.8 \%$ ). Very recently, two uncontrolled studies of reSET-O were published suggesting potential benefits, but due to the possible selection bias and lack of a control group, we did not consider them to add to confidence in the relative effectiveness of the intervention. ${ }^{12,13}$

There were no clinical trials of the Connections app. The trial of the CBT4CBT portion of the app was a fair quality pilot trial that was judged promising but was not definitive, given that the app in this trial did not include the A CHESS intervention that is part of this app, so the degree to which the results are applicable to Connections is highly uncertain.

For the DynamiCare app, there was 1 clinical trial, but it was not solely in the population of interest for this review, and no subgroup results were available in the nonpeerreviewed report of the trial. Thus, we were unable to assess the potential effect of this app in patients with OUD on MAT.

\section{LIMITATIONS OF THE CLINICAL EVIDENCE}

The primary source of limitations in the clinical evidence for these DHTs is the complete lack of peer-reviewed data on the effect of their use for patients with OUD treated with MAT. In addition, the trial designs that demonstrated some efficacy for the behavioral components implemented in the DHTs did not measure outcomes with long enough followup. The minimum follow-up to demonstrate a meaningful effect on adherence would be 6 months, and 12 to 24 months would be more convincing. Finally, no data were reported on key health outcomes that matter to patients, such as ER visits, hospitalizations, return to work, and improved relationships with family and friends.

\section{LONG-TERM COST-EFFECTIVENESS}

Given the dearth of data on Connections and DynamiCare, we only modeled the cost-effectiveness of reSET-O. Where data allowed, the model compared reSET-O as an adjunct to outpatient buprenorphine with outpatient buprenorphine alone. The base-case analysis took a health care system perspective and a 5-year time horizon. We deviated from the ICER Reference Case lifetime time horizon because there was no identified or plausible effect on costs or outcomes beyond the 5-year time horizon.

Despite no evidence on the effects of reSET-O after 12 weeks, our model extrapolated the potential downstream benefits of having a higher percentage of individuals retained on MAT at 12 weeks. At the end of reSET-O completion (12 weeks), the reSET-O and comparator arm in the model discontinued MAT at the same rate of discontinuation observed in the comparator arm of the trial. We used \$1,219 per download as the cost for reSET-O, which was the price provided to us by the manufacturer that was calculated net of rebates, discounts, allowances, and warranty payments. The outcomes of interest included the incremental cost per additional year in MAT and the cost per quality-adjusted life-year (QALY) gained, lifeyears gained, and equal value of life-years gained (evLYG). Full details on ICER's cost-effectiveness analysis and 


\section{TABLE 1 Incremental Cost-Effectiveness Ratios Compared with Best Supportive Care for the Base Case}

\begin{tabular}{l|c|c|c|c}
\hline Intervention & $\begin{array}{c}\text { Incremental } \\
\text { Cost per Life } \\
\text { Year Gained }\end{array}$ & $\begin{array}{c}\text { Incremental } \\
\text { Cost per QALY } \\
\text { Gained }\end{array}$ & $\begin{array}{c}\text { Incremental } \\
\text { Cost per evLYG }\end{array}$ & $\begin{array}{c}\text { Incremental } \\
\text { Cost per } \\
\text { Additional } \\
\text { MAT Year }\end{array}$ \\
\hline reSET-O vs. BSC & $\$ 48,449,000$ & $\$ 121,500$ & $\$ 121,400$ & $\$ 10,000$ \\
\hline
\end{tabular}

$B S C=$ best supportive care including buprenorphine; $e v L Y G=$ equal value life-year gained;

MAT = medication-assisted treatment; $Q A L Y=$ quality-adjusted life-year. model are available on ICER's website at https://icer.org/wp-content/ uploads/2020/08/ICER_DigitalTherapeutics-for-OUD ModelAnalysis-Plan_08032020.pdf and includes a modified societal perspective, given the high societal costs and consequences of OUD.

Table 1 summarizes the costeffectiveness results for reSET-O in patients with OUD. If the assumptions used in the model are true, the incremental cost per QALY gained is $\$ 121,500$, which is within typical costeffectiveness thresholds in the United States. However, as noted earlier, the evidence on long-term retention in MAT is highly uncertain, and the cost per QALY ranged from approximately $\$ 50,000$ to $\$ 500,000$ per QALY over the range of plausible estimates.

\section{LIMITATIONS OF THE COST- EFFECTIVENESS MODEL}

As noted earlier, there is considerable uncertainty about the long-term effectiveness of reSET-O, and nearly all of the major effects on MAT and other outcomes require retention beyond the 12-week duration of the trial evidence. The model's assumptions therefore can be viewed as an optimistic scenario. The base-case findings should therefore be interpreted with caution.

\section{Policy Discussion}

The Midwest Comparative Effectiveness Public Advisory Council (CEPAC) is one of the independent appraisal committees convened by ICER to engage in the public deliberation of the evidence on clinical and cost-effectiveness of health care interventions. The Midwest CEPAC is composed of medical evidence experts, including practicing clinicians, methodologists, and leaders in patient engagement and advocacy. Their deliberation includes input from clinical experts and patient representatives specific to the condition under review, as well as formal comment from manufacturers and the public. A policy roundtable concludes each meeting, during which representatives from insurers and manufacturers join clinical experts and patient representatives to discuss how best to apply the findings of the evidence to clinical practice, insurance coverage, and pricing negotiations.

The ICER report on DHTs for OUD was the subject of a Midwest CEPAC meeting on November 20, 2020. Following the discussion, the panel members deliberated on key questions raised by ICER's report. The results of their votes on the clinical evidence were as follows: (a) the panel voted 10-3 that the clinical evidence was not adequate to demonstrate greater net health benefit for reSET-O compared with best supportive care; (b) the panel voted 13-0 that the evidence was not adequate to demonstrate greater net health benefit for either the Connections app or the Dynamicare app compared with best supportive care.

The CEPAC panel also voted on "other potential benefits" and "contextual considerations" as part of a process intended to signal to policymakers whether there are important considerations when making judgments about long-term value for money not adequately captured in analyses of clinical effectiveness and/ or cost-effectiveness. The results of these votes are shown in Table 2. They highlight several factors beyond the results of cost-effectiveness modeling that the CEPAC panel felt were particularly important for judgments of overall long-term value for money.

As described in ICER's Value Assessment Framework, questions on long-term value for money are subject to a value vote when incremental cost-effectiveness ratios for the interventions of interest are between $\$ 50,000$ and $\$ 175,000$ per QALY in the primary base-case analysis. Eight members of the CEPAC voted that reSET-O at the current discounted price represented low value for money, and 5 members voted that it represented intermediate value. No one voted that it represented high long-term value for money.

The policy roundtable discussion explored how best to translate the evidence and additional considerations into clinical practice and into pricing and insurance coverage policies. The full set of policy recommendations can be found in the Final Evidence Report on the ICER website: https://icer. org/wp-content/uploads/2020/08/ ICER_DHTs_for_OUD_Final_ Evidence_Report_121120-1.pdf. Several key policy recommendations for DHTs follow: 


\section{TABLE 2}

Votes on Other Benefits and Contextual Considerations for the reSET-O App

\begin{tabular}{|c|c|c|}
\hline 1 (Suggests Lower Value) & 2 (Intermediate) & 3 (Suggests Higher Value) \\
\hline $\begin{array}{l}\text { This intervention will not differen- } \\
\text { tially benefit a historically disadvan- } \\
\text { taged or underserved community }\end{array}$ & & $\begin{array}{l}\text { This intervention will differentially } \\
\text { benefit a historically disadvantaged } \\
\text { or underserved community }\end{array}$ \\
\hline 1 vote & 6 votes & 6 votes \\
\hline $\begin{array}{l}\text { Uncertainty or overly favorable } \\
\text { model assumptions creates signifi- } \\
\text { cant risk that base-case cost-effec- } \\
\text { tiveness estimates are too optimistic }\end{array}$ & & $\begin{array}{l}\text { Uncertainty or overly unfavor- } \\
\text { able model assumptions creates } \\
\text { significant risk that base-case } \\
\text { cost-effectiveness estimates are } \\
\text { too pessimistic }\end{array}$ \\
\hline 5 votes & 8 votes & 0 votes \\
\hline $\begin{array}{l}\text { Very similar mechanism of action to } \\
\text { that of other active treatments }\end{array}$ & & $\begin{array}{l}\text { New mechanism of action compared } \\
\text { to that of other active treatments }\end{array}$ \\
\hline 1 vote & 7 votes & 4 votes \\
\hline $\begin{array}{l}\text { Delivery mechanism or relative } \\
\text { complexity of regimen likely to lead } \\
\text { to much lower real-world adherence } \\
\text { and worse outcomes relative to an } \\
\text { active comparator than estimated } \\
\text { from clinical trials }\end{array}$ & & $\begin{array}{l}\text { Delivery mechanism or relative sim- } \\
\text { plicity of regimen likely to result in } \\
\text { much higher real-world adherence } \\
\text { and better outcomes relative to an } \\
\text { active comparator than estimated } \\
\text { from clinical trials }\end{array}$ \\
\hline 2 votes & 9 votes & 2 votes \\
\hline $\begin{array}{l}\text { Will not significantly reduce the } \\
\text { negative impact of the condition } \\
\text { on family and caregivers vs. the } \\
\text { comparator }\end{array}$ & & $\begin{array}{l}\text { Will significantly reduce the negative } \\
\text { impact of the condition on family } \\
\text { and caregivers vs. the comparator }\end{array}$ \\
\hline 1 vote & 9 votes & 3 votes \\
\hline $\begin{array}{l}\text { Will not have a significant impact } \\
\text { on improving return to work and/ } \\
\text { or overall productivity vs. the } \\
\text { comparator }\end{array}$ & & $\begin{array}{l}\text { Will have a significant impact on } \\
\text { improving return to work and/ } \\
\text { or overall productivity vs. the } \\
\text { comparator }\end{array}$ \\
\hline 1 vote & 10 votes & 2 votes \\
\hline
\end{tabular}

1. MAT saves lives and money inside the health system and outside of it. DHTs may be important aids in improving care for many individuals, but it is vital that adequate evidence be generated to evaluate the relative effectiveness of different options so that each person can receive effective treatment tailored to maximize their health. Poor evidence that leads to ineffective use of DHTs represents a health risk to individuals, a financial risk to the health system, and a moral risk for us all that society will fail in its responsibility to use its resources to the greatest effect in combatting an ongoing national epidemic.

2. Manufacturers should provide robust evidence of the clinical effectiveness and broader effect of new DHTs. For DHTs such as those featured in this report that have a function of guiding or enhancing treatment outcomes, a minimum evidence requirement is high-quality observational or quasi-experimental studies with an appropriate comparator and relevant patient outcomes. However, many DHTs should undergo formal evaluation through randomized controlled trials to minimize the risk of bias in trial results.

3. Given the limited evidence supporting the efficacy of DHTs for OUD, alternative payment models, such as guaranteed outcomes, a subscription model, or initial pilot projects may be appropriate if coverage is provided.

4. Manufacturers and researchers should design trials of DHTs to be able to identify potential subgroups of patients who benefit most from a DHT to better target the intervention.

\section{DISCLOSURES}

Funding for this summary was contributed by Arnold Ventures, California Health Care Foundation, The Donaghue Foundation, Harvard Pilgrim Health Care, and Kaiser Foundation Health Plan to the Institute for Clinical and Economic Review (ICER), an independent organization that evaluates the evidence on the value of health care interventions. ICER's annual policy summit is supported by dues from AbbVie, Aetna, America's Health Insurance Plans, Anthem, Alnylam, AstraZeneca, Biogen, Blue Shield of CA, BoehringerIngelheim, Cambia Health Services, CVS, Editas, Evolve Pharmacy, Express Scripts, Genentech/Roche, GlaxoSmithKline, Harvard Pilgrim, Health Care Service Corporation, HealthFirst, Health Partners, Humana, Johnson \& Johnson (Janssen), Kaiser Permanente, LEO Pharma, Mallinckrodt, Merck, Novartis, National Pharmaceutical Council, Pfizer, Premera, Prime Therapeutics, Regeneron, Sanofi, Spark Therapeutics, uniQure, and United Healthcare.

Whittington, Campbell, and Pearson are employed by ICER. Tice reports contracts to his institution, University of California, San Francisco, from ICER during the conduct of this study.

\section{ACKNOWLEDGMENTS}

The authors thank Noemi Fluetsch, Zunelly Odhiambo, Maggie O'Grady, Pamela Bradt, Rick Chapman, Lorenzo Villa Zapata, and Nicholas D. Mendola for their contributions to this report. 


\section{REFERENCES}

1. Kolodny A, Courtwright DT, Hwang CS, et al. The prescription opioid and heroin crisis: a public health approach to an epidemic of addiction. Annu Rev Public Health. 2015;36:559-74.

2. Centers for Disease Control and Prevention. Provisional drug overdose death counts. 2018. Accessed March 4, 2021. https://www.cdc.gov/nchs/nvss/ vsrr/drug-overdose-data.htm

3. U.S. Department of Health and Human Services. National opioid crisis. 2019. Accessed March 4, 2021. http://www.hhs. gov/opioids

4. Centers for Disease Control and Prevention. Overdose deaths accelerating during COVID-19. Updated December 18, 2020. Accessed March 4, 2021. https:// www.cdc.gov/media/releases/2020/ p1218-overdose-deaths-covid-19.html
5. Council of Economic Advisors. The full cost of the opioid crisis: $\$ 2.5$ trillion over four years. October 28, 2019. Accessed March 4, 2021. https://trumpwhitehouse. archives.gov/articles/full-cost-opioidcrisis-2-5-trillion-four-years/

6. Timko C, Schultz NR, Cucciare MA, Vittorio L, Garrison-Diehn C. Retention in medication-assisted treatment for opiate dependence: a systematic review. J Addict Dis. 2016;35(1):22-35.

7. Rash CJ, Stitzer M, Weinstock J. Contingency management: new directions and remaining challenges for an evidencebased intervention. J Subst Abuse Treat. 2017;72:10-18.

8. Fiellin DA, Moore BA, Sullivan LE, et al. Long-term treatment with buprenorphine/naloxone in primary care: results at 2-5 years. Am J Addict. 2008;17(2):116-20.

9. Hser YI, Evans E, Huang D, et al. Long-term outcomes after randomization to buprenorphine/naloxone versus methadone in a multi-site trial. Addiction. 2016;111(4):695-705.
10. Parran TV, Adelman CA, Merkin B, et al. Long-term outcomes of office-based buprenorphine/naloxone maintenance therapy. Drug Alcohol Depend. 2010;106(1):56-60.

11. Christensen DR, Landes RD, Jackson L, et al. Adding an Internet-delivered treatment to an efficacious treatment package for opioid dependence. J Consult Clin Psychol. 2014;82(6):964-72.

12. Velez FF, Colman S, Kauffman L, Ruetsch C, Anastassopoulos K. Realworld reduction in healthcare resource utilization following treatment of opioid use disorder with reSET-O, a novel prescription digital therapeutic. Expert Rev Pharmacoecon Outcomes Res. 2021;21(1):69-76.

13. Maricich YA, Xiong X, Gerwien R, et al. Real-world evidence for a prescription digital therapeutic to treat opioid use disorder. Curr Med Res Opin. 2021;37(2):175-83. 